\title{
FAULT TOLERANCE IN NETWORKED CONTROL SYSTEMS UNDER INTERMITTENT OBSERVATIONS
}

\author{
JEAn-PhILIPPE GEORGES *, Didier THEILliOL*, VinCENT COCQUEMPOT **, \\ JEAN-CHRISTOPHE PONSART *, CHRISTOPHE AUBRUN* \\ * Nancy Research Centre for Automatic Control \\ Nancy University, CNRS, B.P. 70239 F-54506 Vandœuvre les Nancy, France \\ e-mail: didier.theilliol@cran.uhp-nancy.fr \\ ** Automatic Control, Computer Science and Signal Processing Laboratory: LAGIS FRE CNRS 3303 \\ Polytech'Lille, Bât. D, 59655 Villeneuve d'Ascq Cedex, France
}

\begin{abstract}
This paper presents an approach to fault tolerant control based on the sensor masking principle in the case of wireless networked control systems. With wireless transmission, packet losses act as sensor faults. In the presence of such faults, the faulty measurements corrupt directly the behaviour of closed-loop systems. Since the controller aims at cancelling the error between the measurement and its reference input, the real outputs will, in such a networked control system, deviate from the desired value and may drive the system to its physical limitations or even to instability. The proposed method facilitates fault compensation based on an interacting multiple model approach developed in the framework of channel errors or network congestion equivalent to multiple sensors failures. The interacting multiple model method involved in a networked control system provides simultaneously detection and isolation of on-line packet losses, and also performs a suitable state estimation. Based on particular knowledge of packet losses, sensor fault-tolerant controls are obtained by computing a new control law using fault-free estimation of the faulty element to avoid intermittent observations that might develop into failures and to minimize the effects on system performance and safety.
\end{abstract}

Keywords: network congestion, fault-tolerant control, fault diagnosis, networked control system, interacting multiple model.

\section{Introduction}

During the last years, fault-tolerant control has received more and more attention (Blanke et al., 2000). The aim of Fault-Tolerant Control (FTC) is to adjust or to modify on-line the nominal control laws in order to maintain the safety of the operators and the reliability of the processes. The survey paper of Patton (1997) gives the state of the art in the field of fault accommodation. Almost all the methods can be categorised into two groups (Zhang and Jiang, 2008): passive and active approaches.

Passive FTC deals with a presumed set of system component failures considered at the controller design stage. An active FTC system is characterised by an on-line Fault Detection and Isolation (FDI) process and a control reconfiguration mechanism. According to the FDI module, the control reconfiguration mechanism is designed in order to take into account the possibility of fault occurrence. Advanced and sophisticated controllers have been developed with fault accommodation and tolerance capabilities, as, e.g., in the work of Noura et al. (2000). Nowadays, systems tend to be highly distributed, with communication networks being the core structure employed to transport the data. Moreover, there is an increasing trend to employ wireless networks for that role as they support mobility, increase the flexibility and simplify the cabling.

In an industrial plant, severe constraints may apply concerning the Quality of Service (QoS), and possibly the dependability of the system. This is the case for many embedded systems present in medical and industrial applications. The study and design of such applications, called Networked Control Systems (NCSs) as defined by Zhang et al. (2001), has become an important research field. Usually, NCSs are subject to unknown network induced delays and data dropouts. The control issues of NCSs, especially in the case of delays, have attracted the attention of many researchers, with taking into 
account network characteristics (see Tipsuwan and Chow, 2003).

When wireless networks are concerned, the quality of service of the communications can be relatively low compared to with networks. Various communication protocols for wireless networks have been proposed. Their relevance was studied by Decotignie (2002), De Pellegrini et al. (2006) and Willig et al. (2005). Nevertheless, most of the standard wireless protocols remain basically nondeterministic. The main difference between wired and wireless networks comes from the fact that losses cannot be neglected in wireless communications. Decotignie (2002) mentioned that compared with cables, radio transmissions suffer from Bit Error Rates (BERs) that are some orders of magnitude higher. BERs of $10^{-3}$ to $10^{-4}$ are usual whereas in cables one may expect BERs ranging from $10^{-7}$ to $10^{-9}$. Consequently, radio transmission can be easily jammed by perturbed systems. This is especially true for ISM (Instrument, Scientific and Medical) bands. It may completely suppress all communications for long periods of time. A wireless transmission channel is subject to various disturbances which will cause transmission errors. This mostly corresponds to environmental noise and collisions. Although packet losses on a wireless network are intermittent, they might impact the behaviour of closed-loop systems.

In NCSs like sensor networks, in which the communications between the sensors and the controller are ensured thanks to wireless links, this means that losses may occur, and hence measurements can be lost, too. From the controller point of view, the measures are, in such a case, not available, which is the same result as a sensor fault. As a result, an FDI study of systems in which measurements are sent by a wireless channel will have to consider not only sensor faults but also packet losses. In that case, this means that the fault detection system will have to distinguish between a sensor fault and a packet loss. On the controller, it is already possible to add a promptness indicator which will be able to determine if a new measure was received during the sampling time. Promptness indicators were developed for wired industrial networks like WorldFip (CENELEC, 1996). However, it is not sufficient to totally ensure that the last data received were produced (by the sensing task) recently. Due to the asynchronism between the sensing and the communication tasks, a breakdown of the sensing task will not be detected by the previous indicator. This issue might be addressed by a freshness indicator. However, it needs resources and computations which are not always available on sensors.

To the best of our knowledge, theoretical and practical results considering simultaneous on-line detection and isolation of packet losses and also a suitable state estimation are rarely studied. A great amount of effort has been devoted to fault detection of networked control systems with missing measurements (He et al., 2009; Wang et al., 2009; Patan and Uciński, 2008). Some recent works focus their attention on fault detection based on a stochastic variable to describe the dropout or intermittent measurements without (Zhao et al., 2009) or with (Mao et al., 2009) communication time delays. Based on transition matrix probabilities of a packet loss, this paper will hence address active FTC analysis by considering both sensor faults and measurement losses without requiring special resources in sensors.

Xiong and Lam (2007) have recently considered the stabilisation of linear systems with a bounded packet loss under Markovian packet losses assumptions. According to this knowledge, this paper provides an efficient FDI module based on the Interacting Multiple Model (IMM) algorithm (Henk et al., 1988) in order to develop a sensor fault masking method. In this study, the communication between the sensors and the controller will be assumed to be achieved thanks to a wireless network, and it will be also assumed that the delay remains small (compared with the sampling time). Here, the communications between the controller and the actuators are supposed not to be achieved through a network (or at least by a wired network on which both delay and packet losses are neglected).

The paper is organized as follows. In Section 2 a general formulation of the problem is given. Section 3 is devoted to present the IMM algorithm under channel errors or network congestion equivalent to sensor failures models according to a transition matrix probability. According to the FDI algorithm result, a sensor fault masking method is presented in Section 4 based on the faultfree state estimation generated by the interacting multiple model algorithm. A simulation example is given in Section 5 to illustrate the proposed method. Finally, concluding remarks are given in the last section.

\section{Problem statement}

2.1. Packet loss sources. In this paper, the closedcontrol loop is assumed to be achieved through a wireless sensors network. For that purpose, the IEEE 802.15.4 (defined by the IEEE Computer Society (2003) wireless protocol is chosen. This is the protocol used by the ZigBee technology. In contrast to Bluetooth and IEEE 802.11, IEEE 802.15.4 has been specifically developed for applications typical for industrial environments. In our case, the IEEE 802.15 .4 network data rate will be $250 \mathrm{~kb} / \mathrm{s}$ in a single channel within the $2.4 \mathrm{GHz}$ band. This network is expected here to work in the contention access modality only, where access to the shared medium is controlled by means of a distributed CSMA/CA scheme. In such unbeaconed mode, the introduction of random waiting time leads to nondeterminism medium access. Moreover, Decotignie (2002) explains that collisions cannot be detected while sending a message since the power of remote emit- 
ters is much lower than that of the transmitter emission that masks the others. Data packets are hence repaired at the MAC layer on bursty channels like in wireless networks. The error is consequently detected hop-by-hop (and not end-to-end like in TCP), and packet losses are repaired by a packet retransmission protocol like ARQ. However, there is no forwarding error correction coding.

As noticed by De Pellegrini et al. (2006), the radio transmission system is a first cause of losses, particulary in hostile environments, where several types of noise may cause transmission errors. De Pellegrini et al. (2006) showed that these kinds of errors are mostly due to the co-existence of different wireless technologies in a single environment and in the same frequency band. Indeed, the $2.4 \mathrm{GHz}$ band hosts BT, IEEE 802.15.4, IEEE 802.11 , and possibly other systems. This specific issue is addressed by Willig et al. (2005). The second cause of losses deals with the MAC protocol and, specifically, the presence of collisions in the CSMA/CA scheme. Collisions are created by simultaneous access to the medium when the medium is free or because radio communication suffers from the so-called hidden terminal effect.

In the next section, the characterisation of packet losses will be achieved by simulations of an industrial networked control system using the IEEE 802.15.4 MAC protocol.

2.2. Packet loss prediction. In order to be able to isolate a packet loss occurrence, special attention has to be paid to wireless channel models. In the literature, various models have been used. The most popular works in this field are based on the Gilbert/Elliot model (see, for instance, Willig et al., 2002). Various modifications of this model have been then proposed. Used in conjunction with experimental measurements, the Packet Error Rate (PER) in the BAD state might be adjusted in order to achieve an average PER ranging from $10^{-3}$ to $10^{-4}$, as explained by De Pellegrini et al. (2006) and Willig et al. (2002). However, such models do not allow characterising the packet loss ratio dedicated to each communication.

To achieve this objective of per communication packet loss prediction, we propose to use channel noise models in order to simulate bit errors on a wireless communication sketch. Our method is based on experimental measurements achieved on a real plant (an overhead travelling crane presented in Section 5.1). In the work of Cuzzocrea et al. (2008) a measurement survey was carried out in order to draw the map of electromagnetic disturbances induced from the environment and originating from the process equipment itself. An important observation was that the communication is mainly affected by a series of impulses of varying duration and amplitude. These results were then introduced as environmental noise in a global simulation of the NCS by using the TrueTime library (Andersson et al., 2007). This simulation integrates process modelling, closed-loop control, an IEEE 802.15.4 wireless network for communication between sensors and a controller and, finally, environmental noise models.

A promptness indicator was also added on the controller in order to produce packet loss traces. It might be viewed as a healthy indicator of the network. Indeed, the controller maintains a signal per sensor which indicates if a new packet has been received during the last sampling time. This indicator consumes hence few resources so that it might be implemented on a device such as the controller. Based on it, the controller might know if a new value has been received and hence adapt its computations. Figure 1(b) represent the evolution of such indicators when two stations periodically (each $10 \mathrm{~ms}$ ) send data on an IEEE 802.15.4 network facing an impulsional noise. Figure 1(a) shows the associated packet losses according to the network point of view.
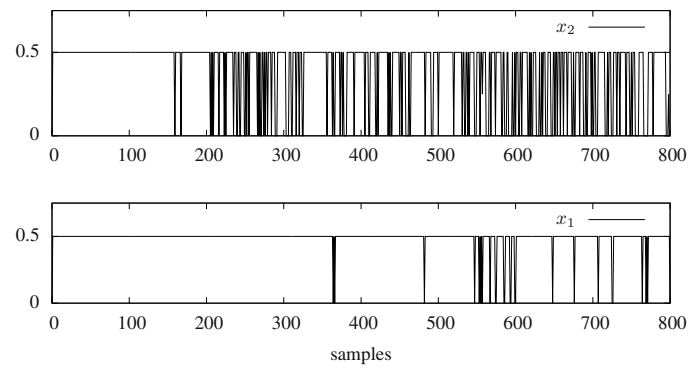

(a)
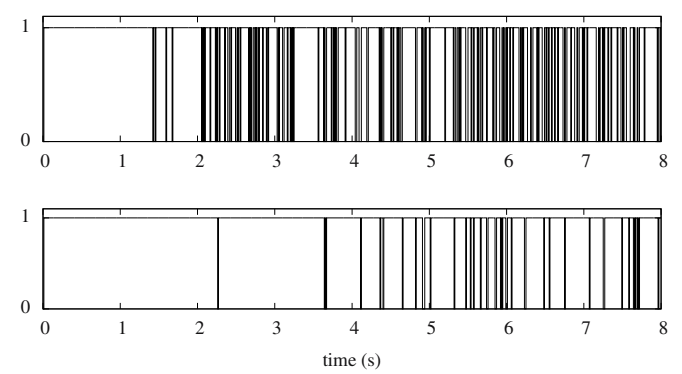

(b)

Fig. 1. Simulation of packet losses on a sample network: packet losses showing the quality of the transmission of the two measurements on the wireless network $(0.5$ indicates a successful transmission, 0.25 a retransmission due to a collision and 0 a packet loss (a), associated promptness indicators (1 indicates a successful transmission during the last period and 0 a packet loss ) (b).

The signal is assumed to be able to detect if the signal level in the receiving node is larger than the receiver signal threshold. The Signal-to-Noise Ratio (SNR) is then computed by taking into account the environmental noise and all other transmissions (which act as background noise). A packet is assumed to be correctly received only if the percentage of bit errors is lower than the error coding 
threshold. Hence, packet losses will occur if the noise it much larger than the signal level. Since the longer the distance between the sender and the receiver, the higher the SNR sensible to the environmental noise, and packet losses are specific for each communication as shown in Fig. 1. Moreover, additional losses are possible if collisions occur, i.e., if the medium access is not able to send the packet. This corresponds to the 0.25 signal level in Fig. 1(a). Also note that Fig. 1(b) might highlight situations when network induced delays are larger than the sampling time such that no measures were received.

The simulations show that it is possible to dynamically determine the packet losses ratio (also called the failure rate) for each communication. Relatively to loss sources, packet loss rates were observed as time varying (and intermittent). Simulations enable us to classify three situations (modes): no losses, intermittent losses for a given traffic and a special case corresponding to a wireless network out of order. The promptness computation algorithm is then extended in order to dynamically determine the probability to move from one situation to another. Assume four modes $j$ such that $j=0$ stands for the case when all communications are successful, $j=1$ and $j=2$ when only the first (and, respectively, the second) communication is unsuccessful, and $j=3$ denotes the case where all communications are unsuccessful. Considering simulations of Fig. 11 the final transition probabilities are given by

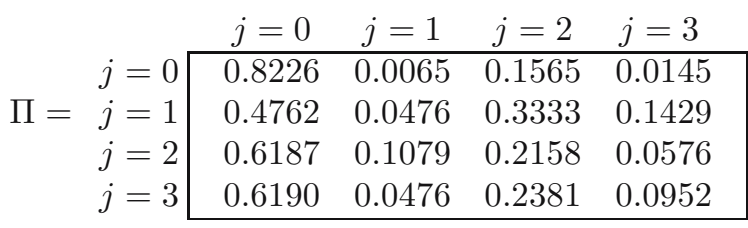

The value in the matrix $\Pi$ represents the probability to move from one mode to another. By comparing $\Pi_{2,2}$ and $\Pi_{0,2}$, it can be noticed that bursty noise is taken into account. This matrix might be computed at each sampling time thanks the promptness indicators. At this point, the problem might be hence resumed as follows: Detect and isolate measurements errors (channel errors or packet losses) on the controller equivalent to sensor failures and, finally, reconfigure the control.

\section{Fault detection, isolation and estimation module}

In the spirit of fault diagnosis, the basic idea of the approach is to reconstruct the state of the system from subsets of measurements in the presence of an intermittent package loss which corresponds to a channel error or network congestion and causes a straight packet loss. The objective is to build a bank of filters where each filter is based on a "faulty" model under the knowledge of a transition probability matrix from one mode to another. In order to solve this problem, let us consider a linear system in the stochastic case defined as

$$
\left\{\begin{aligned}
x(k+1) & =A x(k)+B u(k)+\xi(k), \\
y(k) & =C x(k)+\eta(k),
\end{aligned}\right.
$$

where $x \in \mathbb{R}^{n}$ is the state vector, $u \in \mathbb{R}^{p}$ is the input vector, and $y \in \mathbb{R}^{m}$ is the output vector. Here $\xi \in \mathbb{R}^{n}$ (respectively, $\eta \in \mathbb{R}^{m}$ ) represents the plant noise vector (resp., the measurement noise vector). $A, B$ and $C$ are constant matrices with appropriate dimensions.

According to the dynamic behavior of a packet loss, which is similar to a fault on a sensor, sensor failures are considered to represent network failures in the fault diagnosis approach. Each $j$-th faulty output can be written as

$$
y_{j}^{f}(k)=\beta_{j} y_{j}(k),
$$

where $y_{j}$ and $y_{j}^{f}$ denote the $j$-th nominal and faulty sensor, respectively, with $\beta_{j} \in[0,1]$. A sensor failure corresponds to $\beta_{j}=0$.

As proposed by Zhang and Li (1998) in the multi model framework with the interacting multiple model algorithm, such a linear system in the presence of faults/failures can be considered a stochastic hybrid system. The system mode sequence is then an indirectly hidden Markov chain where a transition probability matrix $\Pi$ from Mode $i$ to Mode $j$ is a design parameter. Consequently, a sensor failure is modelled by setting to zero the appropriate column of the output matrix $C$ :

$$
\left\{\begin{aligned}
x(k+1) & =A x(k)+B u(k)+\xi(k), \\
y(k) & =\left[C+F_{j}\right] x(k)+\eta(k),
\end{aligned}\right.
$$

where the matrix $F_{j}$ contains zero elements except that the $j$-th row is taken to be the negative of the $j$-th row of $C$.

In this paper, simultaneous sensor "failures" are assumed to occur. Consequently, $q+1=2^{m}$ (with $y \in \mathbb{R}^{m}$ ) models should be considered in the set of possible failure modes. Here the number $q$ of rows $(q \leq m)$ in matrix $F_{j}$ can be the negative of the corresponding row of $C$. Compared with the works of Zhang and Li (1998) or Theilliol et al. (2008), which extended the interaction multiple model algorithm to a nonlinear system, this paper takes into account not only a single fault, but a set of faults designed by the matrix $\bar{C}_{j}=\left[C+F_{j}\right]$ defining the faulty model. It attempts to extend the interaction multiple model algorithm, developed by Zhang and Li (1998), to the network problem. The four steps of the interacting multiple model algorithm are briefly outlined below.

Interaction. $\forall j \in 0, \ldots, q$, a predicted mode probability is calculated:

$$
\mu^{j-}=\sum_{t=0}^{q} \pi_{t}^{j} \mu^{t}(k-1),
$$


where $\mu^{t}(k-1)$ represents the conditional mode probability associated with the $j$-th faulty sensor and $\pi_{t}^{j}$ is an element of the transition probability matrix $\Pi$ from one mode to another. We have

$$
\begin{aligned}
& P_{j}(k-1 \mid k-1) \\
& =\sum_{t=0}^{q} P_{t}(k-1 \mid k-1) \mu^{t}(k) \\
& \quad+\sum_{t=0}^{q}\left[\widehat{x}(k-1 \mid k-1)-\widehat{x}_{j}(k-1 \mid k-1)\right] \\
& \quad \times\left[\widehat{x}(k-1 \mid k-1)-\widehat{x}_{j}(k-1 \mid k-1)\right] \mu^{t}(k) .
\end{aligned}
$$

A mixed state is estimated by

$$
\widehat{x}_{j}(k-1 \mid k-1)=\sum_{t=0}^{q} \mu^{t}(k) \widehat{x}_{t}(k-1 \mid k-1),
$$

with the associated covariance given in (5), where

$$
\mu^{t}(k)=\frac{\pi_{t}^{j} \mu^{t}(k-1)}{\mu^{j-}}
$$

defines the mixing probability.

Filtering. $\forall j \in 0, \ldots, q$, a predicted state is calculated as follows:

$$
\widehat{x}_{j}(k \mid k-1)=A \widehat{x}_{j}(k-1 \mid k-1)+B u(k-1),
$$

with the associated covariance equivalent to

$$
P_{j}(k \mid k-1)=A P_{j}(k-1 \mid k-1) A+Q^{j} .
$$

The measurement residual and the filter gain can be computed as

$$
\begin{gathered}
r_{j}(k)=y_{j}(k)-\bar{C}_{j} \widehat{x}_{j}(k \mid k-1), \\
K_{j}(k)=P_{j}(k \mid k-1)\left(\bar{C}_{j}\right)^{T}\left(\Omega_{j}\right)^{-1},
\end{gathered}
$$

with $\Omega_{j}=\bar{C}_{j} P_{j}(k \mid k-1)\left(\bar{C}_{j}\right)^{T}+R_{j}$.

In this way, a filtered state can be estimated as

$$
\left.\widehat{x}_{j}(k \mid k)=\widehat{x}_{j}(k \mid k-1)+K_{j}(k)\right) r_{j}(k),
$$

with the following associated covariance matrix:

$$
P_{j}(k \mid k)=P_{j}(k \mid k-1)-K_{j}(k) \Omega_{j}\left(K_{j}(k)\right)^{T} .
$$

Mode probability calculation. $\forall j \in 0, \ldots, q$, a classical likelihood function based on residuals distribution is determined as

$$
\ell_{j}(k)=\frac{\exp \left(-0.5 r_{j}(k)\left(\Omega_{j}\right)^{-} 1\left(r_{j}(k)\right)^{T}\right)}{\sqrt{2 \pi \Omega_{j}}} .
$$

According to this function, a mode probability can be calculated as follows:

$$
\mu^{j}(k)=\frac{\mu^{j-} \ell_{j}(k)}{\sum_{t=0}^{q} \mu^{t-} \ell_{t}(k)} .
$$

It should highlighted that the mode probabilities $\mu^{j}$ provide an indication of the active mode at each sampling period $k$. Mode probabilities can be used to isolate the faulty sensor. Moreover, they can be used in a supervision scheme in order to provide operators with information about the occurrence of a possible failure.

Combination. Based on the previous steps, the state estimate is computed by a weighted sum of the following form:

$$
\widehat{x}(k \mid k)=\sum_{j=0}^{q} \mu^{j}(k) \widehat{x}_{j}(k \mid k),
$$

used in the performance index evaluation. Moreover, a fault-free estimation can be established as $\widehat{y}(k \mid k)=$ $C \widehat{x}(k \mid k)$ used in a control law: the control law is becoming "robust" against failures and faults, as proposed in the next paragraph.

\section{Sensor fault masking}

Around an operating point, the following discrete state space representation is considered:

$$
\left\{\begin{aligned}
x(k+1) & =A x(k)+B u(k), \\
y(k) & =C x(k), \\
z(k) & =C_{r} x(k),
\end{aligned}\right.
$$

where $A \in \mathbb{R}^{n \times n}, B \in \mathbb{R}^{n \times p}, C \in \mathbb{R}^{m \times n}$ and $C_{r} \in$ $\mathbb{R}^{h \times n}$ are the state, the control, the output and the output reference matrices, respectively. Here $x \in \mathbb{R}^{n}$ is the state space, $u \in \mathbb{R}^{p}$ is the input vector, and $z \in \mathbb{R}^{h}$ corresponds to the measured output vector while $y \in \mathbb{R}^{n}$ represents the system outputs that will track the reference inputs $r \in \mathbb{R}^{h}$.

In order to maintain controllability, the number of outputs $h$ that can track a reference input vector $r$ cannot exceed the number of control inputs $p \geq h$. For this study, a Linear Quadratic Regulator (LQR) is considered to design the controller of a multi-input and multi-output system. Since the feedback control can only guarantee the stability and dynamic behaviour of the closed-loop system, a complementary controller is required to track the reference input vector $r$ in the sense that the steady-state response is

$$
\lim _{k \rightarrow+\infty} y=r .
$$

Various techniques have been developed to achieve steady-state tracking of the reference input. Among them, a feedforward control law based on a command generator tracker (Zhang and Jiang, 2002) can be considered,

$$
u^{\text {nom }}(k)=-K_{\text {forward }}^{\text {nom }} r(k)-K_{\text {feedback }}^{\text {nom }}, \widehat{x}(k),
$$


where $K_{\text {forward }}^{\text {nom }}$ is synthesized on the basis of the closedloop model-following principle and $\widehat{x}(k)$ represents the state estimate obtained classically, for instance, by means of a Kalman filter.

However, in the presence of sensor faults, the faulty measurements corrupt directly the closed-loop behaviour. Moreover, the controller aims at cancelling the error between the measurement and its reference input. But in this case, the real outputs are different from the desired value and may drive the system to its physical limitations or even to instability. Sensor fault-tolerant control can be obtained by computing a new control law using fault-free estimation of the faulty element to avoid faults that could develop into failures and to minimize the effects on the system performance as defined in Eqn. (16).

From the control point of view, sensor fault-tolerant control does not require any modifications of the control law and is also called "sensor masking", as suggested by $\mathrm{Wu}$ et al. (2006). The only requirement is that the "estimator" provides an accurate estimate of the system output after a network fault occurs. Fault diagnosis in the developed strategy is of paramount importance to compensate for these faults and to preserve the system performances. Moreover, it should be highlighted that the model probabilities (Eqn. (15)) provide an indication of the mode in effect at any time.

\section{Application: An overhead travelling crane}

5.1. Process description. Figure 2 shows the synoptic view of the plant. A metal bar (length: $1.2 \mathrm{~m}$, weight: $1 \mathrm{~kg}$ ) is positioned along a $6 \mathrm{~m}$ length axis by two linear motors $(12 \mathrm{~kg} / \mathrm{each})$. The maximum speed is equal to $4 \mathrm{~m} / \mathrm{s}$ with a maximum acceleration of $4 \mathrm{~g}$. The goal of

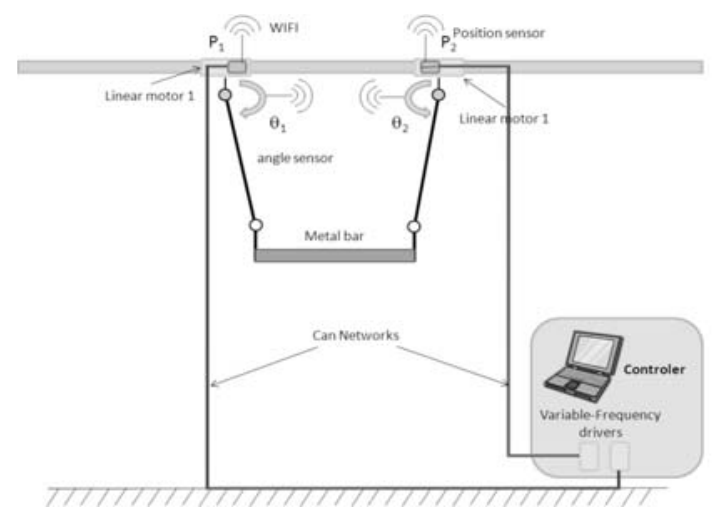

Fig. 2. Synoptic of the overhead travelling crane benchmark.

the control is to shift the metal bar to a reference position under the constraint to keep the bar horizontal. The measurements used correspond to angle measurements at the motor $\left(\theta_{1}\right.$ and $\left.\theta_{2}\right)$ and position measurements provided by variable-frequency drives $\left(p_{1}\right.$ and $\left.p_{2}\right)$. The transmissions of the measurements between the sensors and the controller are achieved thanks to a wireless network based on the IEEE 802.15.4 protocol as illustrated in Fig. 3

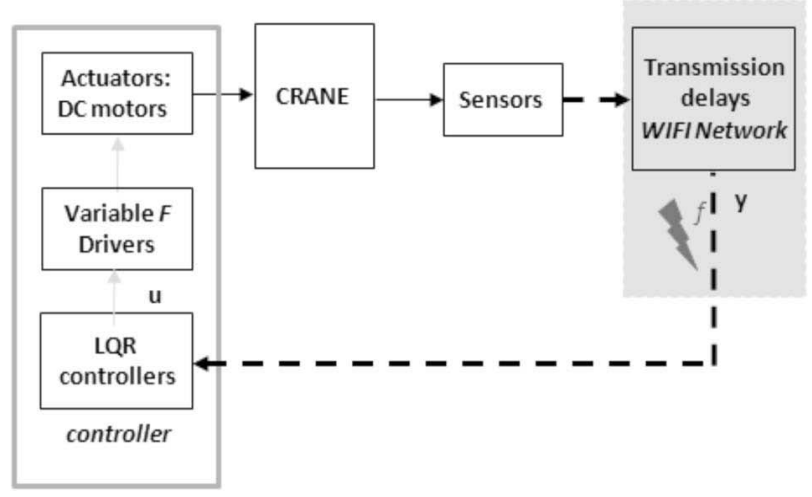

Fig. 3. Networked control overhead travelling crane scheme.

As the two positions are measured by sensors, the output vector is $y=\left[\begin{array}{ll}p_{1} & p_{2}\end{array}\right]^{T}$. The control input vector is associated with the motor drive $u=\left[\begin{array}{ll}u_{1} & u_{2}\end{array}\right]^{T}$. The purpose is to control the system around an operating position. Under the assumption that the $\theta_{1}$ and $\theta_{2}$ angles of the metal bar are equal to zero, a discrete state space representation can be obtained using a Taylor expansion with a sampling period $T_{s}=0.1 \mathrm{~s}$ with the state vector $x$ equal to

$$
x=\left[\begin{array}{llllllll}
p_{1} & p_{2} & \theta_{1} & \theta_{2} & \dot{p}_{1} & \dot{p}_{2} & \dot{\theta}_{1} & \dot{\theta}_{2}
\end{array}\right]^{T} .
$$

These outputs are controlled using the multivariable control law described previously. The control matrix pair of the augmented plant is controllable, and the nominal tracking control law, designed by an LQR technique, provides feedback/forward gain matrices $\left(K_{\text {feedback }}^{\text {nom }} / K_{\text {forward }}^{\text {nom }}\right)$ with $\operatorname{diag}(Q)=$ $\left[\begin{array}{llllllll}10 & 10 & 1 & 1 & 0 & 0 & 10 & 10\end{array}\right]^{T}$ fixed by the noise level. Four faulty models have been considered: a faultfree case $(j=0)$, a network problem associated with $p_{1}(j=1)$ and $p_{2}(j=2)$, and a network out of order, i.e., simultaneously $p_{1}$ and $p_{2}(j=3)$.

The results shown in the following figures are responses with respect to set-point changes. In the simulation, a Gaussian noise is added to each output signal. The reference inputs correspond to step changes for $p_{1}$, and $p_{2}$ which excited the whole behaviour of the nonlinear system.

Firstly, the validation of the tracking control is shown in Fig. 4, where step responses are considered for a range of $20 \mathrm{~s}$. Reference inputs $r$ are step changes for $p_{1}$ and $p_{2}$. The dynamic responses demonstrate that a tracker is synthesised correctly. As illustrated in Fig. 4, the $\theta_{1}$ and $\theta_{2}$ angles of the metal bar are closed which corresponds to the assumption. 


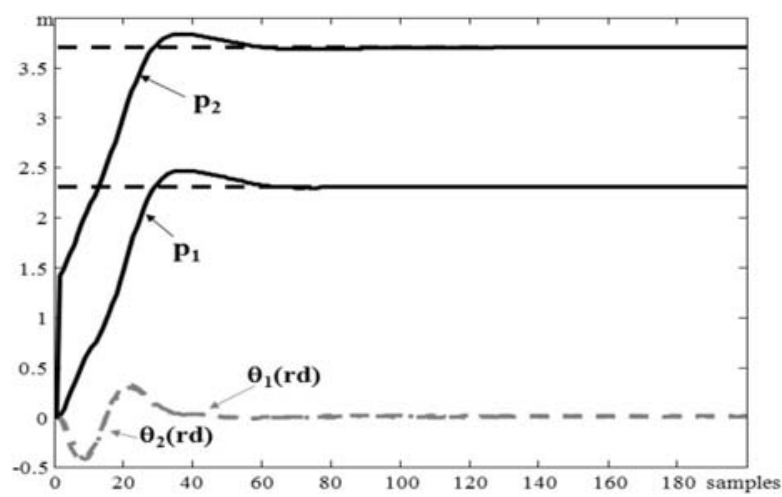

Fig. 4. System outputs controlled by a classical LQR control law in a fault-free case.

As presented in Section 2.2, different network configurations were simulated in order to identify the transition probability matrix $\Pi$. In the first simulation, the controller is assumed to be fixed in coordinates $(0,1)$, i.e., in front of the first motor at the start of the simulation. Moreover, the maximum number of retransmissions in the case of a collision is limited to 3 . It is also assumed that the motors are sending simultaneously the position measurements $p_{1}$ and $p_{2}$ :

$$
\begin{array}{rlcccc}
\multicolumn{1}{c}{} & j=0 & j=1 & j=2 & j=3 \\
\cline { 3 - 6 } \Pi_{a}=0 & 0.8017 & 0.0353 & 0.1008 & 0.0622 \\
j=1 & 0.5349 & 0.1163 & 0.3256 & 0.0233 \\
j=2 & 0.5652 & 0.1217 & 0.2609 & 0.0522 \\
j=3 & 0.6042 & 0.0625 & 0.2500 & 0.0833
\end{array}
$$

In the second case, it is now assumed that the position measurements are not sent simultaneously. This leads to a new matrix defined by

$$
\begin{array}{rlcccc} 
& & j=0 & j=1 & j=2 & j=3 \\
\cline { 3 - 6 } \Pi_{b}=0 & 0.8226 & 0.0065 & 0.1565 & 0.0145 \\
j=1 & 0.4762 & 0.0476 & 0.3333 & 0.1429 \\
j=2 & 0.6187 & 0.1079 & 0.2158 & 0.0576 \\
j=3 & 0.6190 & 0.0476 & 0.2381 & 0.0952
\end{array}
$$

Compared with the first case, fewer packet losses occurs. This is due to the fact that, in the first case, since the transmission of the position measurements is synchronised, collisions occur, which leads to packet losses. By adding an offset, this kind of loss is eliminated so that the probability to move in the fault-free case $(j=0)$ is increased. The impact of the collisions is also linked to the number of retransmission trials. Indeed, the following matrix was obtained by limiting the retransmission threshold to 1 :

$$
\begin{array}{rlcccl|}
\multicolumn{1}{c}{} & j=0 & j=1 & j=2 & j=3 \\
\cline { 3 - 6 } \Pi_{c}=0.7847 & 0.0053 & 0.1993 & 0.0107 \\
j=1 & 0.3571 & 0.0714 & 0.5000 & 0.0714 \\
j=2 & 0.5317 & 0.0341 & 0.3902 & 0.0439 \\
j=3 & 0.3000 & 0.1500 & 0.3500 & 0.2000 \\
\cline { 3 - 6 } & & &
\end{array}
$$

Here more packet losses occur since there are fewer possibilities to successfully transmit the position measurements.

Finally, it might be noticed in the previous matrix that the transition probabilities are relatively different between the cases $j=1$ and $j=2$, i.e., the position measurement of the first and the second motor. This is related to the position of the motors $\left(p_{1}, 0\right)$ and $\left(p_{2}, 0\right)$ during the simulation and the fixed position of the controller. Indeed, Fig. 4 shows that the motors are moving away from the controller $(0,1)$ so that the relevant SNR is more and more sensitive to the environmental noise. And since the second motor set point is higher than that of the first one, the second motor is already more sensible to this noise as shown by Fig. 1. Yet, if the controller is now mobile so that it follows the metal bar moving, a new matrix might be obtained,

$$
\begin{array}{rlcccc|} 
& & j=0 & j=1 & j=2 & j=3 \\
\cline { 2 - 6 } \Pi_{d}= & 0.9974 & 0 & 0.0013 & 0.0013 \\
j=1 & 1.0000 & 0 & 0 & 0 \\
j=2 & 1.0000 & 0 & 0 & 0 \\
j=3 & 1.0000 & 0 & 0 & 0 \\
\cline { 2 - 6 } & &
\end{array}
$$

Here fewer packet losses occur since the distance is significantly decreased so that the impact of the noise is also decreased.

To conclude, a transition probability matrix $\Pi$ might be dynamically defined. However, this matrix remains only valid for a given set of network and plant parameters. For instance, the sampling period was fixed according to the dynamics of the plant in order to guarantee the stability of the closed-loop. The transmission period of the measurements $\theta$ and $p$ along the network has been defined according to this sampling period. In order to take into account packet losses, the Shannon theorem should be used and adapted to the transmission period so that, by sending more packets, measurements will finally be computed on the controller. However, if the transmission period decreases, this might be problematic in a nondeterministic network like IEEE 802.15.4 since the percentage of packet losses due to collisions will increase.

In this paper, the reconfiguration method will be illustrated according to the case identified by the matrix $\Pi_{b}$. It might also be noticed that only position measurement packet losses are taken into account-it is assumed that no packet losses appear for the transmission of control inputs. Moreover, this paper does not focus on the impact of network induced delays on the quality of control (interested readers might consult the work of Tipsuwan and Chow (2003)).

5.2. Results and comments. The consequence of an intermittent loss of a package is considered on the first and second channels dedicated to deliver sensor measure- 
ments. As illustrated in Fig. 5, packet losses increase with the distance between the controller and the motors.
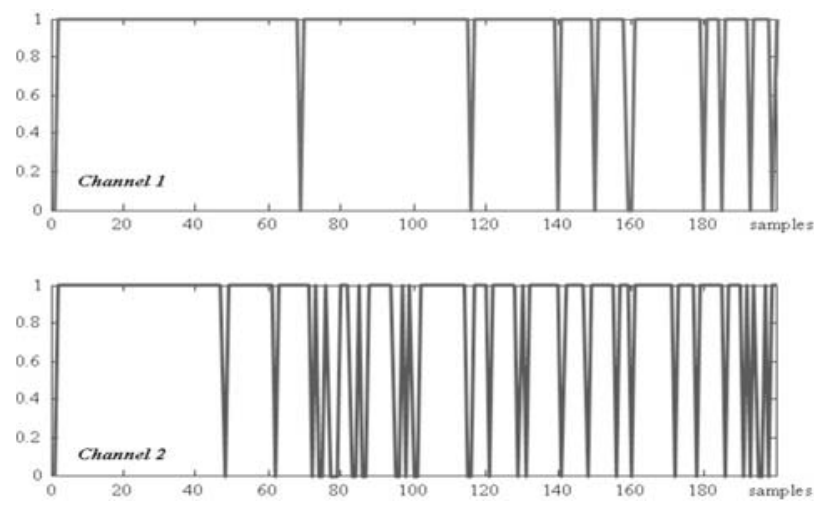

Fig. 5. Promptness indicators of packet losses ( 1 indicates a successful transmission during the last period and 0 a packet loss).

The control law tries to cancel the static error created by the corrupted output: all sensors deliver a value equal to zero. Consequently, the real output is different from the reference input and the control law is different from its nominal value. As presented by Sinopoli et al. (2004), the closed-loop system is unstable (see Fig. 6). Figure 7

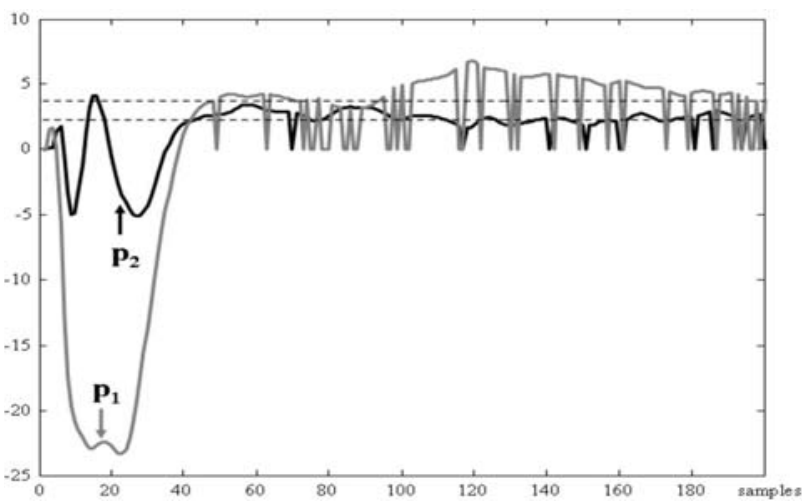

Fig. 6. System outputs controlled by classical LQR control with an intermittent loss of a package.

presents the dynamic evolution of the mode probabilities $\mu^{j}$. When a packet is lost, the observation obtained by the receiver is equal to zero and the mode probability $\mu^{0}$ is down to zero. Compared with the promptness indicators illustrated in Fig. 5, Fig. 7 shows clearly the capabilities of the interacting multiple model algorithms to detect and isolate faults. Without network congestion, the "faultfree" model is always close to dynamic evolution of the promptness indicators (equal to 1 ). Otherwise, the mode probability $\mu^{3}(k)$ presents some abrupt variations due to the simultaneous network problem on both sensors. According to these probabilities, it is possible to detect and isolate the fault.
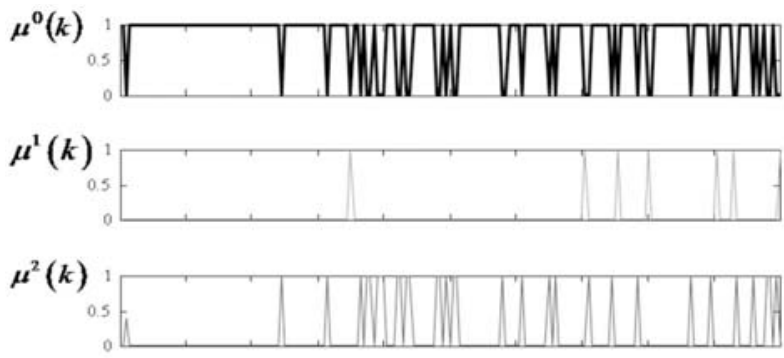

$\mu^{3}(k)$

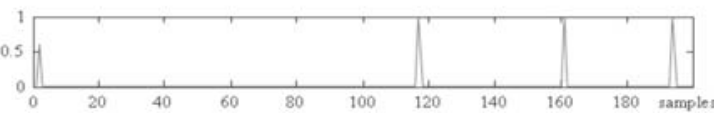

Fig. 7. Mode probabilities evolution with an intermittent loss of a package.

Moreover, based on a suitable model probability estimate, the state estimate, defined in (16), is not corrupted by an intermittent loss of a package. As illustrated in Figs. 8and 9 , with the sensor fault-tolerant control method the real levels follow the reference inputs $r$ close to the nominal case.

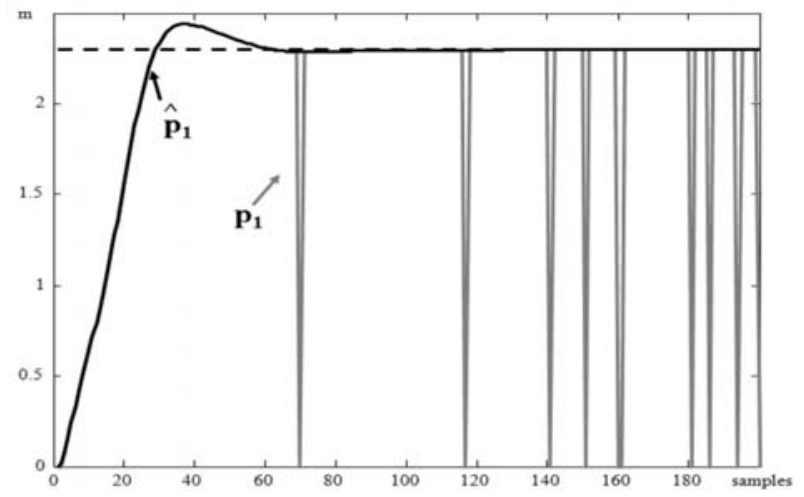

Fig. 8. Estimated and measured system output $p_{1}$ controlled by an FTC scheme with an intermittent loss of a package.

\section{Conclusion}

In this paper, an approach was proposed to tackle the impact of packet losses on FDI/FTC design of a networked control system. A particular kind of NCS consisting of a closed-loop control system integrating a wireless sensor network was considered. Focusing only on packet losses (delays were not studied here), it was shown that packet losses might lead to additional kinds of sensor faults which can impact on the system stability. The developed idea is therefore that the FDI/FTC system has to be adapted to packet loss characteristics, especially to the intermittent one. An FDI/FTC design based on the interacting multiple model approach algorithm based on the transition probability matrix was proposed in order to 


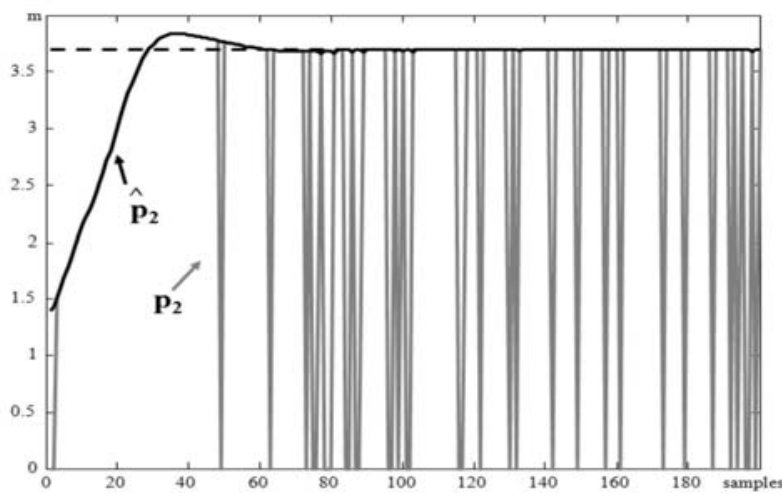

Fig. 9. Estimated and measured system output $p_{2}$ controlled by an FTC scheme with an intermittent loss of a package.

minimize the effects of packet losses on the system performance and safety. Future works should consider sensitivity analysis of the developed method against the uncertainty of failure rates. Moreover, in order to consider a more general case such as problem of time delay, future works will be extended to the network control systems with wireless communication between the controller and the actuators.

\section{Acknowledgment}

This work was supported in part by the CPER MISN SECTAF project (2007-2012, Lorraine, France).

\section{References}

Andersson, M., Henriksson, D. and Cervin, A. (2007). Truetime 1.5: Reference Manual, Department of Automatic Control, Lund Institute of Technology, Lund.

Blanke, M., Frei, C., Kraus, F., Patton, R. and Staroswiecki, M. (2000). What is fault-tolerant control?, IFAC Safeprocess'2000, Symposium Budapest, Hungary, Vol. 1, pp. $40-51$.

CENELEC (1996). Fieldbus. Vol. 1: P-net, Vol. 2: Profibus, Vol. 3: Worlfip, European Standard EN50170.

Cuzzocrea, C., Dandache, A., Georges, J.-P., Jean, P., Monteiro, F., Theilliol, D. and Yamé, J. (2008). Analysis of wireless transmissions QoS relatively to the dependability of a networked control system, 23rd IAR Workshop on Advanced Control and Diagnosis, Coventry, UK, pp. 210-215.

De Pellegrini, F., Miorandi, D., Vitturi, S. and Zanella, A. (2006). On the use of wireless networks at low level of factory automation systems, IEEE Transactions on Industrial Informatics 2(2): 129-143.

Decotignie, J.-D. (2002). Wireless fieldbusses-A survey of issues and solutions, 15th IFAC Triennal World Congress, Barcelona, Spain.

He, X., Wang, Z. and Zhou, D. (2009). Robust fault detection for networked systems with communication delay and data missing, Automatica 45(11): 2634-2639.
Henk, A., Bloom, O. and Bar-Shalom, Y. (1988). The interacting multiple model algorithm for systems with Markovian switching coefficients, IEEE Transactions on Automatic Control 33(8): 780-783.

IEEE Computer Society (2003). IEEE standard for information technology, Telecommunications and information exchange between systems, Local and metropolitan area networks, Specific requirements, Part 15.4: Wireless medium access control (mac) and physical layer (phy) specifications for low-rate wireless personal area networks (lrwpans), IEEE Std 802.15.4-2003.

Mao, Z., Jiang, B. and Shi, P. (2009). Fault detection for a class of nonlinear networked control systems, International Journal of Adaptive Control and Signal Processing 24(7): 610-622.

Noura, H., Sauter, D., Hamelin, F. and Theilliol, D. (2000). Fault-tolerant control in dynamic systems: Application to a winding machine, IEEE Control Systems Magazine 20(1): 33-49.

Patan, M. and Uciński, D. (2008). Configuring a sensor network for fault detection in distributed parameter systems, International Journal of Applied Mathematics and Computer Science 18(4): 513-524, DOI: 10.2478/v10006-0080045-4.

Patton, R. (1997). Fault-tolerant control: The 1997 situation, IFAC Symposium Safeprocess'97, Kingston Upon Hull, $U K$, Vol. 2, pp. 1033-1055.

Sinopoli, B., Schenato, L., Franceschetti, M., Poolla, K., Jordan, M. and Sastry, S. (2004). Kalman filtering with intermittent observations, IEEE Transactions on Automatic Control 49(9): 1453-1464.

Theilliol, D., Rodrigues, M. and Ponsart, J. (2008). Fault diagnosis and accommodation design for nonlinear systems described by interpolated LTI models, 16th Mediterranean Conference on Control and Automation, Ajaccio, France, pp. 267-273.

Tipsuwan, Y. and Chow, M.-Y. (2003). Control methodologies in networked control systems, Control Engineering Practice 11(10): 1099-1111.

Wang, Y., Ding, S., Ye, H., Wei, L., Zhang, P. and Wang, G. (2009). Fault detection of networked control systems with packet based periodic communication, International Journal of Adaptive Control and Signal Processing 23(8): 682698.

Willig, A., Kubisch, M., Hoene, C. and Wolisz, A. (2002). Measurements of a wireless link in an industrial environment using an IEEE 802.11-compliant physical layer, IEEE Transactions on Industrial Electronics 49(6): 12651282.

Willig, A., Matheus, K. and Wolisz, A. (2005). Wireless technology in industrial networks, Proceedings of the IEEE 93(6): 1130-1151.

Wu, E., Thavamani, S., Zhang, Y. and Blanke, M. (2006). Sensor fault masking of a ship propulsion, Control Engineering Practice 14(11): 1337-1345. 
Xiong, J. and Lam, J. (2007). Stabilization of linear systems over networks with bounded packet loss, Automatica 43(1): 8087.

Zhang, W., Branicky, S. and Phillips, S. (2001). Stability of networked control systems, IEEE Control Systems Magazine 21(1): 84-89.

Zhang, Y. and Jiang, J. (2002). Active fault-tolerant control system against partial actuator failures, IEE Proceedings: Control Theory and Applications 149(1): 95-104.

Zhang, Y. and Jiang, J. (2008). Bibliographical review on reconfigurable fault-tolerant control systems, Annual Reviews in Control 32(2): 229-252.

Zhang, Y. and Li, X. R. (1998). Detection and diagnosis of sensors and actuators failures using IMM estimator, IEEE Transactions on Aerospace and Electronic Systems 34(4): 1293-1313.

Zhao, Y., Lam, Y. and Gao, H. (2009). Fault detection for fuzzy systems with intermittent measurements, International Journal of Adaptive Control and Signal Processing 17(2): 298-410.

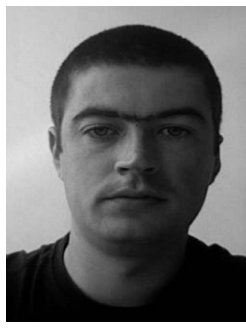

Jean-Philippe Georges received the Ph.D degree in automatic control (network engineering) from Henri Poincar University, Nancy 1, France, in 2005. In 2006 he worked as a researcher with the Laboratory of Process Control and Automation, Aalto University, Finland. He is currently an associate professor with the Research Centre for Automatic Control of Nancy at Nancy University, France. His research interests are in computer networks and networked control systems. His current works are focused on evaluating the performance of wired and wireless networks (especially in the field of network calculus theory) with real-time constraints. He aims at identifying efficient and reliable communication for control and diagnosis systems.

Didier Theilliol received the $\mathrm{Ph} . \mathrm{D}$. degree in control engineering from Nancy University (France) in 1993. Since 2004, he has been a full professor at the Research Centre for Automatic Control of Nancy (CRAN) at Nancy University, where he co-ordinates and leads national, European and international R\&D projects in steel industries, wastewater treatment plant, or the aerospace domain. His current research interests include model-based fault diagnosis method synthesis and active fault-tolerant control system design for LTI, LPV, multi-linear systems. He is the chair of the intelligent control and diagnosis working group, where various French and German research teams are involved. He has published over 150 journal and conference papers.

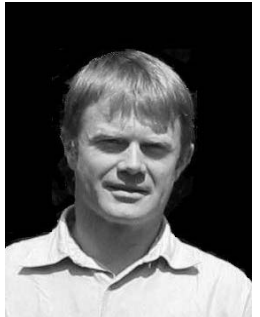

Vincent Cocquempot received the Ph.D. degree in automatic control from the Lille University of Sciences and Technologies in 1993. He is currently a full professor in automatic control and computer science at the Technological University Institute (Institut Universitaire de Technologies) of Lille, France. He is a researcher of the LAGIS-CNRS FRE3303: Automatic Control, Computer Science and Signal Processing Laboratory at Lille 1 University and the head of the team on fault tolerant systems in this laboratory. His research interests include robust on-line Fault Detection and Isolation (FDI) for uncertain dynamical nonlinear systems and Fault Tolerant Control (FTC) for Hybrid Dynamical Systems (HDSs).

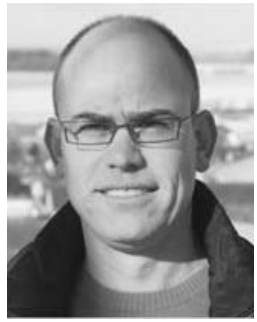

Jean-Christophe Ponsart received his Ph.D. in 1996 from the University of Savoie in Annecy, France, in nonlinear control of magnetic suspensions and its digital implementation aspects. In 1997, he participated in the design and implementation of real-time controllers with a digital signal processor architecture for an industrial company. He has been with the Research Center for Automatic Control of Nancy (CRAN), France, since 1998 as an assistant professor. His current interests include fault diagnosis and fault-tolerant control, in particular for LPV systems and with the application to a winding machine.

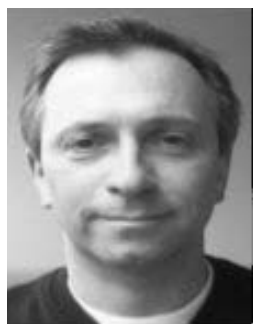

Christophe Aubrun received a Ph.D. in control engineering from the University of Nancy, France, in 1992 . He is currently a member of the Research Centre for Automatic Control of Nancy (CRAN). Since 2005 he has been a professor in the Department of Electrical Engineering at the Institute of Technology, University of Nancy. He has been involved in many projects with industry as well as European projects. His research interests lie in complex systems diagnosis and fault tolerant control areas with particular applications to water treatment processes and networked control systems.

Received: 15 September 2010 Revised: 8 April 2011 